\title{
Conservation of statistical results under the reduction of pair-contact interactions to solvation interactions
}

\author{
N. Hamedani Radja*†, R. R. Farzami ${ }^{\dagger}$, and M. R. Ejtehadi ${ }^{* \dagger \dagger}$ \\ ${ }^{\dagger}$ Department of Physics, Sharif University of Technology, \\ P.O.Box 11365-9161, Tehran, Iran. \\ ${ }^{\ddagger}$ Institute for studies in Theoretical Physics and Mathematics (IPM), \\ P.O.Box 19395-5531, Tehran, Iran.
}

\begin{abstract}
We show that the hydrophobicity of sequences is the leading term in Miyazawa-Jernigan interactions. Being the source of additive (solvation) terms in pair-contact interactions, they were used to reduce the energy parameters while resulting in a clear vector manipulation of energy. The reduced (additive) potential performs considerably successful in predicting the statistical properties of arbitrary structures. The evaluated designabilities of the structures by both models are highly correlated. Suggesting geometrically non-degenerate vectors (structures) as protein-like structures, the additive model is a powerful tool for protein design. Moreover, a crossing point in the log-linear diagram of designability-ranking shows that about $\frac{1}{e}$ of the structures have designabilities above the average, independent on the used model.
\end{abstract}

\footnotetext{
* To whom correspondence may be addressed. E-mail: nima@mehr.sharif.edu or ejtehadi@sharif.edu
} 


\section{INTRODUCTION}

The challenge to understand the different aspects of the protein folding phenomenon has been brought further by means of different disciplines, specially after the classic contribution

by Anfinsen [1]. The extreme complexity of the problem due to the numerous number of interacting atoms has made the study of the problem practically impossible in the atomic scales. The simplified coarse-grained models introduce a feasible way to understand significant macroscopic characteristics of the macromolecules without any reference to the atomic details. However, a relatively impeccable estimation of the effective inter-residue interactions is necessary for such models in order to yield reasonable results.

Although it is believed that the solubility of different residues in the water environment (that is, a single-body effective solvation potential) plays the most significant role in the folding process, the most commonly used class of potentials in the folding problem is the class of two-body interactions e.g. the potential introduced by Miyazawa and Jernigan (MJ) [2]. Introducing an effective contact distance and paying particular attention to the contacts between different residues in a protein's native structure, they reached an estimation of the effective inter-residue interactions by means of a statistical method [2]. The contact energy parameters $M_{i j}$ form a $20 \times 20$ symmetric matrix, namely the MJ matrix $(\mathbf{M})$, presenting 210 independent contact energy parameters between 20 known amino acids. This matrix has been modified later by considering a larger database of proteins [3] and through some self consistent iterative procedures [4, 5, 6]. Although the MJ parameters are not particularly successful in the study of the folding dynamics and mechanism, they predict the groundstate configuration from decoys quite fine [6] .

Analyzing the eigenvalues of MJ matrix, Li et al. have shown that its elements can be approximated quite fine by considering only 22 parameters instead of the original 210 parameters 7]. That is 20 energy parameters, $q$, one residue independent mixing energy parameter, $\gamma$, and a collapsing energy parameter, $E_{c}$, which is physically irrelevant in the energy spectrum of the structures sharing the same compactness. The pair-contact energy between two residues having energy parameter $q$ and $q^{\prime}$ is given by:

$$
E_{q q^{\prime}}=-\left(E_{c}+q+q^{\prime}+\gamma q q^{\prime}\right)
$$

in their model. They argued that the energy parameters ( $q$ 's) are measures of hydrophobicity of residues and their distribution supports simplified two-letters Hydrophobic-Polar (HP) 
model [8]. The authors [7] suggested the HP energy parameters as 1, 0 and 0.3 for $q_{H}, q_{P}$ and $\gamma$ respectively, in an arbitrary energy unit. Furthermore, they have shown that there always exist a set of highly designable structures which are reasonably better candidates as groundstate structures compared to the other configurations, regardless of using the energy parameters either from the HP model [9] or the 20-letters model (MJ energy parameters) [10].

In a recent work 11] it has been shown that most commonly used pair-contact potentials can be divided to two different class of small and large $\gamma$ values. Setting $\gamma=0$ in Eq. (11), we are left with the additive part of the contact potential energy which may be treated as a solvation-like model [12, 13]. In this reduced model, the pair-contact energy of a given protein with $N$ residues in a given configuration may be represented using vectorial notation in a compact fashion:

$$
\begin{aligned}
E\left(\left\{q_{i}\right\},\left\{\vec{r}_{i}\right\}\right) & =\frac{-1}{2} \sum_{i, j}^{N}\left(q_{i}+q_{j}\right) C_{i j} \\
& =-\sum_{i, j}^{N} q_{i} C_{i j} \\
& =-\vec{q} \cdot \vec{v}
\end{aligned}
$$

where $i$ and $j$ are residue's indexes along the chain, $\vec{r}_{i}$ is the position of residue $i$ and $\mathbf{C}$ is the structure's contact matrix. The element $C_{i j}$ is 1 if the distance between the corresponding non-chemically bonded pair in the given structure is smaller than a cut-off radius, $r_{c}$, and is zero otherwise. The vector $\vec{v}$, defined as $v_{i}=\sum_{j=1}^{N} C_{i i}$, is a one dimensional projection of the contact matrix and is called the contact vector [12, 14]. The components of this integer vector are simply the number of contacting neighbors for corresponding residues, regardless to their types. Although the reduced model decreases the computational cost as it reduces matrix products to vector products, this presentation carries noticeably less information compared to the contact matrix representation. The vectors are generally degenerate and any of them may correspond to $g$ different contact matrices. The case in which $g=1$, the vector is non-degenerate and there is a one to one relationship with its corresponding matrix. Recent studies show that the three-dimensional structure is reproducible using onedimensional vector representation for some small proteins [15, 16] which suggest that the contact vectors of protein-like structures have to be non-degenerate.

Consistent with many arguments about the central role of hydrophobic forces in the protein folding problem [17, 18, 19], it has been shown that in the case of two-letters (HP) 
lattice model, the additive (solvation) terms of the potential carry enough information to replicate the energy spectrum of sequences in the configuration space 12]. It has also been shown that non-degenerate contact vectors $(g=1)$ are noticed in parallel to highly designable structures as well as large energy gaps between the groundstates and the first excited states of native proteins [12, 20].

In this study, we show that in the case of 20-letters model, the additive part of MJ matrix carries enough information to manifest highly designable structures, by enumerating all chain configurations in a two dimensional square lattice model up to a length of 36 . Although the applicability of the additive potential instead of MJ potential is not very promising in coming up with the same groundstate structure of a randomly given sequence, its performance is appealing on protein-like sequences.

\section{THE MODEL}

We consider the MJ96 interaction matrix (M) (introduced by Miyazawa and Jernigan at 1996 [3]) as the reference pair-contact interaction matrix between all different types of the residues. The additive potential defined as:

$$
E_{i j}=q_{i}+q_{j}
$$

will be regarded as the first-order approximation of the interaction matrix in which indices $i$ and $j$ (going from 1 to 20) refer to the residue types. Turning back to the approximation made in [7], one can identify the additive potential as the $\gamma=0$ limit of Eq. (11) which may still hold the general behavior of the original model. A similar procedure has been done in the case of the two-letters HP model [12]. The other constant parameter, $E_{C}$, is absorbed into the residue dependent parameters.

The root mean square distance of the two matrices, defined as RMSD = $\sqrt{\sum_{i j}\left(M_{i j}-E_{i j}\right)^{2}}$ has been taken as a measure of the similarity between them. Therefore, a set of additive parameters ( $q$ 's) minimizing the RMSD of the two matrices will theoretically mimic the original inter-residue interactions (MJ matrix). Minimizing RMSD, simply gives q's as:

$$
q_{i}=\langle M\rangle_{i}-\frac{1}{2}\langle M\rangle
$$

where the first term is the average of the strength of interactions between $i$ th amino acid 
and all other ones (including itself) while the second term is a residue independent value, equal to the half of the mean of interactions between all different residue types which may be described as a tendency for proteins to fold into compact structures. For the structures with the same compactness (having the same number of contacts), it causes an irrelevant shift in energy spectrum.

To find the sensitivity of low-energy states to the model interaction energies, we enumerated all maximally compact structures having different lengths of $L=16,20,30,36$. We also studied the whole structures with $L=14,16$ to be sure that the compactness restriction does not affect the conclusions. Working in complete configuration space of larger chains is not feasible in a reasonable time. The time limitation also restricted us to consider only two-dimensional structures. Among all structures which are identical by geometrical symmetries, we chose only one.

Because of the large number of possible sequences, we restricted our evaluation to an ensemble of random sequences, followed by search for their groundstates and first excited states in the structures space. To be more specific, in the case of $L=36$ we sampled $2.4 \times 10^{7}$ different sequences among $20^{36} \simeq 10^{47}$ possible sequences and $10^{7}$ for other cases. This amount of the samples yield to valid statistical results as there was no significant impact on the results even after ignoring half of these samples.

Starting with the MJ96 model as the reference potential, we searched the conformation space for the groundstate and the first excited state of each of the sampled sequences using the pair-contact interactions. Moreover, we evaluated the energy of these two structures using the additive potential. A similar search in the conformation space for the groundstate and the first excited state of each sequence is followed using the additive potential. We did the same comparison with the MJ85 pair-interactions [2] and also for its additive part.

\section{RESULTS}

\section{A. The D matrix}

It is well believed that the hydrophobicity of the residues plays a very important role in the folding process. However, there is no unique scale for measuring this characteristic. Although the different scales agree with each other, they are strongly sensitive to the type of 
the experiment (Table【). As it was mentioned before, the additive potential parameters ( $q$ 's) extract the contribution of hydrophobicity in MJ matrix. Therefore, they may be regarded as a new measure of hydrophobicity. We have compared them with the experimental scales and also with the q parameters, found by Li et al 7] in Table [. The upper half of the table represents the correlation coefficient $(r)$ between hydrophobicity scales where the lower half is dedicated to the corresponding p-values (a measure of significance of the statistics). The table shows a significant correlation between the additive part of MJ matrix and the other hydrophobicity scales. This correlation has been observed formerly by Chan 21] in a reverse way, i.e he noticed a correlation between the elements of MJ matrix and the sum of the hydrophobicity of the corresponding amino acids.

The reconstructed interaction matrix, Eq.(3), is quite similar to the original MJ matrix. The elements of these two matrices have been compared with each other in Figure 1, showing a strong correlation of $r=0.982$ which is even stronger than the correlation between the different revisions of the MJ matrix (0.973) (introduced in 1985 [2] and 1996 [3]). It means that the difference between the additive matrix and the reference one is in the range of statistical errors. Interestingly, the additive parts of the two MJ matrices correlate better than the matrices themselves ( $r=0.979$ in this case).

Figure 2 is a graphical representation of the matrices using a gray-scale intensity plot, i.e darker elements correspond to stronger interactions. As one can conclude, the matrices are quite similar to each other and the relative difference of each element is small, compared to the element itself. Roughly speaking, the left graph (additive) is the faded version of the right one (MJ) in Figure 2. Therefore, we can consider the differences as perturbative terms to the additive matrix. This result agrees with the conclusion of [12] where parameter $\gamma$ was introduced as a perturbation to the HP model.

We define the difference matrix as:

$$
D_{i j}=M_{i j}-\left(q_{i}+q_{j}\right)
$$

We have sketched the distribution of the elements of the matrix D in Figure 3 in order to see how large they are. Except a few large elements, the difference of the two matrices is small, compared to $k_{\mathrm{B}} T$. The energy of a sequence in a specific structure consists of the interaction energy of all of its contacts, meaning that this cumulative error may be not as negligible as of a single contact. Based on the Central Limit Theorem, the mean and the 
standard deviation of the sum of $N$ randomly chosen elements of $\mathbf{D}$ are larger than mean and the standard deviation of its elements, by factors $N$ and $\sqrt{N}$ respectively. For example, highly compact $2 D$ structures with 36 residues on a square lattice have 25 contacts between the residues. The above discussion suggests that employing the additive matrix instead of the MJ matrix is plausible, together with a random energy with a zero mean and a standard deviation of $1.40 k_{\mathrm{B}} T$. This random deviation is large enough to make the groundstates unstable. However, we will show that the chain correlations and the correlated shifts in the energy spectra considerably moderate the above naïve estimation.

\section{B. The statistical properties of structures with different lengths}

As mentioned before, the vector representation of configurations is a tricky method for decreasing the CPU run time as it reduces the matrix calculations to vector calculations. It is also responsible for some new degeneracies. In other words, in the vector model, all of

the structures belonging to a vector with $g>1$ have the same energy. Thus, none of them can correspond to an unique groundstate. Table II reports the number of contact matrices, contact vectors, structures with unique contact vectors and also the average of $g$ for different lengths, and Figure 4 shows the first three of these parameters in a log-log plot. Although the number of vectors with $g=1$ is increasing with length, its ratio to the total number of structures is decreasing. Thus, the average value of $g$ is an increasing function of length, implying that the probability of being a protein-like structure is smaller than that of short lengths for larger structures.

\section{Protein-like sequences}

Searching for the groundstate of any given sequence using the MJ energy parameters, we will deal with one of the following scenarios:

1. It does not have a global minimum energy configuration: It is not a protein-like sequence and is not a matter of our interest.

2. It has a global minimum energy configuration but the structure corresponds to a vector with $g>1$ : There are $g-1$ other configurations which have the same form in vector 
representation. Thus this sequence does not have a unique groundstate using the vector (additive) model. It has been shown that in the case of HP lattice model,

such sequences are of little interest and do not behave as protein-like sequences [12]. More careful considerations are required to conclude the same statement for 20-letters models.

3. It has a global minimum energy configuration and the structure corresponds to a vector with $g=1$ : In the HP model, such structures are usually highly designable. Folding in highly designable structures, such sequences will be called protein-like sequences.

In order to find the frequency of occurrence of each of the above scenarios, we have studied a set of randomly chosen sequences. Table 11 shows the ratio of sequences belonging to each scenario. The average energy gaps, the energy differences between the groundstates and the first excited states, are also reported for different scenarios. The sequences belonging to the third scenario have a considerably larger energy gap which is yet another observation relating the protein-like structures to those having non-degenerate vector representations $(g=1)$. The probability of occurrence of the first scenario remains almost constant for different lengths but the probability of belonging to the second and third scenarios increases and decreases with their lengths, respectively. It means that fewer sequences have the chance to fold in a structure with $g=1$, i.e. those which are claimed to be the protein-like sequences.

\section{A comparison between the observable parameters of the reference and the} additive potential

The aim of this section is to use the additive potential as an approximation to the reference pair-contact potential. Thus, a relevant question is how successful it is in finding the groundstate structures. Our investigation shows that using the MJ96 potential as the reference and for the structures with length of 36 , about $33 \%$ of sequences belonging to the third scenario fold in the same structures, once we employ the additive approximation. Apparently, it seems that the additive approximation is not promising in the prediction of the groundstates. However, comparing the two versions of the MJ potential (MJ96 and MJ85), the overlap between the groundstates of these sequences is found to be only $43 \%$.

It should be noted that in this comparison, we only consider the third scenario struc- 
tures as the others are not candidates for being groundstates, according to the additive model. Comparing the two version of the MJ potentials, we are also able to look at the same groundstates belonging to the second scenario. We found a similarity of about $36 \%$ in this case which is less than the same study of the third scenario. This conclusion is consistent with our previous observation that these structures possess smaller energy gaps. This is another fact that lets us consider structures with non-degenerate contact vectors as reasonable candidates for the groundstates of protein-like structures.

Although the additive potential is not successful in evaluating the same groundstates found by MJ potential, we may still investigate its capability in finding the general properties of the structures such as the spectrum of designability. The designability is defined as the number of sequences folding into a specific structure (see for example [9]). Figure 5 is a comparison of the designabilities of compact structures evaluated by the additive model $\left(n_{s}^{\prime}\right)$ with the evaluations of the MJ model $\left(n_{s}\right)$ for $L=36$. As mentioned before, we have ignored the structures with $g>1$. Concluding from the corresponding figure, the parameters mentioned above strongly correlate with each other $(r=0.956)$. The correlation coefficient for compact structures with $L=16,20,30$ are $0.996,0.992,0.959$, respectively. Considering the whole structure space for $L=14,16$, the designabilities still show strong correlations of 0.997 and 0.996 respectively. Designabilities of structures in two different versions of the MJ potential (MJ96 and MJ85) also agree with each other $(r=0.994$, Figure 5 ), meaning that the relative designability of structures is considerably insensitive to small perturbations in interactions.

Additionally, it is also notable that the first few highly designable structures are similar in both models, which means that the additive potential is able to indicate highly designable structures successfully. It should be noted that yielding to these structures is considerably more expensive using the reference pair-contact potential. The conservation of designability order has been previously reported in the case of two-letters model [20]. Looking at the difference between the designabilities of structures in the additive and reference potentials, (Figure 60) we notice that it is highly peeked near zero, implying that it is possible to indicate the designability of structures using the additive model with a small relative error in most cases. However the tail of the histogram in Figure [6] warns for the possibility of evaluating substantially different designabilities. A closer look at Figure 5 ensures us that the order (rank) of designability is more conserved, specially for highly designable structures. 
Therefore, one can conclude that the additive potential is more successful in the study of the statistical characteristics of the structures than single designs.

\section{E. A crossing point in designability-ranking graphs}

We have also observed that regardless of the used potential, designability obeys a universal law in terms of ranking. Figure 7 reflects the designability of all structures as a function of their rank for different potentials, including three versions of the MJ interactions introduced by Miyazawa and Jernigan in 1985, 1996 and 1999 and also the additive potential extracted from MJ96. In all of these models, the graphs are linear in a semi-log plot (Figure [7-a), specially for highly designable structures. Assuming that

$$
r=A e^{-\lambda n_{s}}
$$

where $r$ and $n_{s}$ are the rank of a specific structure and its designability respectively and $\lambda$ is a model-dependent free parameter.

The normalization factor $A$ may be determined by noting that the summation over designability of all structures equals to the number of studied sequences, $S$. That is,

$$
\begin{aligned}
S=\sum_{r=1}^{N} n_{s}(r) & =\frac{1}{\lambda} \sum_{r=1}^{N}(\ln A-\ln r), \\
& =\frac{1}{\lambda}(N \ln A-\ln (N !)),
\end{aligned}
$$

where $N$ is the number of structures. Solving the last equation for $A$, employing the Stirling's approximation and substituting it into Eq. (6), we finally reach to:

$$
r=N e^{\frac{\lambda S}{N}-1-\lambda n_{s}}=\frac{N}{e} e^{-\lambda\left(n_{s}-\bar{n}_{s}\right)} .
$$

Where $\bar{n}_{s}=S / N$ denotes the average designability of the structures.

Equation (8) introduces a fix point on $n_{s}=\bar{n}_{s}$, meaning that the rank of structure with $n_{s}=\frac{S}{N}$ is independent on the model and is equal to $\frac{N}{e}$ (see Figure 7). By rescaling the rankings with respect to the total number of structures (which is different for additive and non-additive potentials) and subtracting $\bar{n}_{s}$ from the designabilities, the fixed point appears more visible (Figure [7-b). The same feature has been previously observed for two-, fourand infinite-letters models [22]. 


\section{F. The first excited state energy gap}

It is believed that the native states of most proteins are stable against thermal fluctuations and mutations. This stability is a result of large gap between the free energy of the native states and the first excited states, which is a characteristic of the protein-like sequences. If we approximate the difference of free energy with the energy gap of states, we expect a positive correlation between the averages of the energy gaps of the sequences which fold into a specific structure, $\langle\Delta\rangle$, and the designability of the corresponding structure.

Comparing the average energy gap of different structures in the additive and the reference potential yields to quite similar diagrams (Figure 8), although the energy gaps in additive model are slightly smaller than those of MJ model. The difference of the number of points of the additive and MJ graphs is a result of ignoring structures having $g>1$ in additive model. There is a jump in the energy gap diagram of short sequences which may be used to distinguish low and high designable structures from each other (e.g. length 16 in Figure 8 8 and b). This transition becomes smoother for sequences with larger length (e.g. length 36 in Figure 8-c and d). The jump in energy gap is a distinguished characteristic of finite size structures which is more visible in the models with less free parameters (such as the HP model) than the complicated models (such as MJ reference potential) [9, 12]. However, the highly designable structures possess a larger energy gap in average, regardless of the used model.

Figure 9 reports the probability of finding the same structure as groundstate in different models as a function of energy gap, $\Delta$. The probability increases monotonically as a function of $\Delta$ which means that deeper groundstates of the reference potential have a higher chance to be groundstates in the additive model too.

Marking the groundstate and the first excited structure of each sequence in the MJ potential model and comparing the folding energy difference in this model, $\Delta$, with the energy difference in the additive model, $\Delta^{\prime}$, we reach to a meaningful correlation between them. If these structures had been chosen randomly, we would have expected a Gaussian distribution for $\Delta-\Delta^{\prime}$ centered in origin. However, this difference has a non-zero mean

centered at $0.11 k_{\mathrm{B}} T$ (Figure 10) for these models. The small tendency of these structures to fold in the same groundstate is associated to their smaller energy differences in the additive model. 


\section{G. Protein design using additive potential}

Designing a protein can be described as finding a sequence, $\sigma$, which maximizes the Boltzmann probability of finding the protein in a specific configuration, $S^{*}$. Therefore, one must maximize:

$$
P=\frac{e^{\frac{-H\left(\sigma, S^{*}\right)}{k_{\mathrm{B}} T}}}{\sum e^{\frac{-H\left(\sigma, S^{i}\right)}{k_{\mathrm{B}} T}}},
$$

with respect to $\sigma$, where $H\left(\sigma, S^{*}\right)$ is the folding energy of $\sigma$ in structure $S$. The additive potential may be used as a first approximation in protein design. We have studied the ability of the additive model to design sequences for all 69 maximally compact $2 D$ square lattice structures with $L=16$. This attempt failed for 24 of them, basically due to the degeneracy of their contact vectors $(g>1)$, together with 6 other structures with $g=1$. In these cases, the sequence which maximizes the probability has degenerate groundstate. For the remaining 39 structures, we could successfully design a sequence to fold in the additive potential model. Moreover, the evaluated groundstate structures remains unchanged under the application of MJ potential. The energy gap of these sequences varies in the range $2.90 k_{\mathrm{B}} T$ to $8.56 k_{\mathrm{B}} T$ and the mean value of the gaps is equal to $5.91 k_{\mathrm{B}} T$.

\section{CONCLUSION}

We have shown that the additive part of the pair-contact interactions between amino acids

(introduced by Miyazawa and Jernigan [3] ) essentially represents the main characteristics of the reference interactions. Taking the additive part as the leading term, we have shown that the deviations may be considered as a first-order perturbation to the reference interactions. The additive (solvation) model introduces less energy parameters while reduces the encoding of structures from a matrix form to a vector form.

Considering the MJ96 potential as the reference potential, we have investigated the conservation of the groundstates of the structures under employing the additive model. The groundstates of about one fourth of sequences are conserved in the case of $6 \times 6$ structures. This number is improved to one third if we consider only non-degenerate $(g=1)$ structures.

The additive potential is quite successful in predicting the statistical properties of the structures. The designability of the structures evaluated by the reduced model is highly correlated to the reference model. The additive model successfully indicates the highly 
designable structures (evaluated by the reference potential), all of them being among $g=1$ structures. This observation suggests that non-degenerate vectors (structures) extensively resemble the protein-like structures. Sequence design for such structures is also successful using the additive model.

Taking the other version of MJ matrix (MJ85) as another perturbation to the MJ96 results in a same magnitude of deviation, although the computation is much cheaper using the additive model.

We have also observed a crossing point in the log-linear diagram of designability ranking,

showing that about $\frac{1}{e}$ of the structures have designabilities above the average, independent on the used model.

\section{Acknowledgments}

We would like to thank M. Babadi and B. Mehmani for carefully reading the manuscript and useful comments.

[1] C. B. Anfinsen, Science 181, 223 (1973).

[2] S. Miyazawa and R. L. Jernigan, Macromolecules 18, 534 (1985).

[3] S. Miyazawa and R. L. Jernigan, J. Mol. Biol. 256, 623 (1996).

[4] L. A. Mirny and E. I. Shakhnovich, J. Mol. Biol. 264, 1164 (1996).

[5] H. S. Chan and K. A. Dill, Proteins 24, 335 (1996).

[6] S. Miyazawa and R. L. Jernigan, Proteins 34, 49 (1999).

[7] H. Li, C. Tang, and N. S. Wingreen, Phys. Rev. Lett. 79, 765 (1997).

[8] H. S. Chan and K. A. Dill, J. Chem. Phys. 95, 3775 (1991).

[9] H. Li, R. Helling, C. Tang, and N. S. Wingreen, Science 273, 666 (1996).

[10] H. Li, C. Tang, and N. S. Wingreen, Proteins 49, 403 (2002).

[11] P. Pokarowski, A. Kloczkowski, R. L. Jernigan, N. S. Kothari, M. Pokarowska, and A. Kolinski, Proteins 59, 49 (2005).

[12] M. R. Ejtehadi, N. Hamedani, H. Seyed-Allaei, V. Shahrezaei, and M. Yahyanejad, Phys. Rev. E 57, 3298 (1998). 
[13] M. Skorobogatiy, H. Guo, and M. J. Zuckermann, Macromolecules 30, 3403 (1997).

[14] A. Kabakçioğlu, I. Kanter, M. Vendruscolo, and E. Domany, Phys. Rev. E 65, 041904 (2002).

[15] M. Porto, U. Bastolla, H. E. Roman, and M. Vendruscolo, Phys. rev. Lett. 92, 218101 (2004).

[16] A. R. Kinjo and K. Nishikawa, Bioinformatics 21, 2167 (2005).

[17] C. Tanford, Science 200, 1012 (1978).

[18] G. D. Rose, A. R. Geselowitz, G. J. Lesser, R. H. Lee, and M. H. Zehfus, Science 229, 834 (1985).

[19] G. J. Lesser and G. D. Rose, Protiens 8, 6 (1990).

[20] V. Shahrezaei and M. R. Ejtehadi, J. Chem. Phys. 113, 6437 (2000).

[21] H. S. Chan, in Encyclopedia of life sciences, edited by D. Atkins (Nature Publishing Group, London, U.K., 2001).

[22] N. E. Buchler and R. A. Goldstein, Proteins 34, 113 (1999).

[23] The references for hydrophobicity scales presented in the table are: (HS1) D. Eisenberg, E. Schwarz, M. Komaromy, and R. Wall, J. Mol. Biol. 179, 125 (1984), (HS2) J. Kyte and R. F. Doolittle, J. Mol. Biol. 157, 105 (1982), (HS3) W. C. Wimley and S. H. White, Nature Struct. Biol. 3, 842 (1996), (HS4) W. C. Wimley, T. P. Creamer, and S. H. White, Biochemistry 35 5109 (1996), (HS5) S. H. White and W. C. Wimley, Biochim. Biophys. Acta 1376, 339 (1998), (HS6) S. H. White and W. C. Wimley, Annu. Rev. Biophys. Biomol. Struc. 28, 319 (1999), (HS7) D. R. DeVido, J. G. Dorsey, H. S. Chan, and K. A. Dill, J. Phys. Chem. B 102, 7272 (1998), (HS8) J. L. Fauchère and V. Pliška, Eur. J. Med. Chem.18, 369 (1983). 
TABLE I: Correlation between different hydrophobicity scales [23] (The upper half represent the correlation coefficients and the lower half is the p-value multiplied by $10^{5}$ )

\begin{tabular}{llcccccccccc}
\hline \hline Hyd. scale & ADD96 & ADD85 & HS1 & HS2 & HS3 & HS4 & HS5 & HS6 & HS7 & HS8 & Li et al \\
\hline ADD96 & - & 0.979 & 0.790 & 0.830 & 0.758 & 0.818 & 0.724 & 0.759 & 0.836 & 0.914 & 0.996 \\
ADD85 & 1 & - & 0.783 & 0.806 & 0.758 & 0.807 & 0.744 & 0.739 & 0.834 & 0.918 & 0.979 \\
HS1 & 41 & 46 & - & 0.879 & 0.686 & 0.760 & 0.668 & 0.724 & 0.776 & 0.875 & 0.808 \\
HS2 & 21 & 31 & 9 & - & 0.587 & 0.723 & 0.452 & 0.751 & 0.705 & 0.810 & 0.857 \\
HS3 & 70 & 70 & 214 & 864 & - & 0.925 & 0.787 & 0.721 & 0.718 & 0.835 & 0.759 \\
HS4 & 25 & 31 & 67 & 123 & 4 & - & 0.655 & 0.930 & 0.801 & 0.887 & 0.828 \\
HS5 & 121 & 88 & 281 & 4324 & 43 & 338 & - & 0.434 & 0.754 & 0.780 & 0.698 \\
HS6 & 68 & 95 & 121 & 78 & 127 & 3 & 5230 & - & 0.767 & 0.811 & 0.776 \\
HS7 & 19 & 19 & 52 & 161 & 132 & 34 & 75 & 60 & - & 0.870 & 0.826 \\
HS8 & 4 & 4 & 9 & 29 & 19 & 7 & 49 & 29 & 10 & - & 0.920 \\
Li et al & 1 & 1 & 30 & 13 & 68 & 21 & 181 & 52 & 22 & 4 & - \\
\hline \hline
\end{tabular}

TABLE II: The number of contact matrices and contact vectors of maximally compact structures on $2 \mathrm{D}$ square lattices

\begin{tabular}{lcccc}
\hline \hline Length & 16 & 20 & 30 & 36 \\
\hline Structures & 69 & 503 & 13498 & 57337 \\
Vectors & 56 & 398 & 9514 & 35662 \\
Vectors with g=1 & 45 & 309 & 6819 & 23921 \\
Average of g & 1.23 & 1.26 & 1.42 & 1.61 \\
\hline \hline
\end{tabular}

TABLE III: Probability of occurrence of each scenario with their average energy gap

\begin{tabular}{llccc}
\hline \hline Length & 16 & 20 & 30 & 36 \\
\hline Probability of first scenario & $3.8 \%$ & $3.2 \%$ & $3.1 \%$ & $3.3 \%$ \\
Probability of second scenario & $23.4 \%$ & $24.7 \%$ & $33.1 \%$ & $37.4 \%$ \\
Probability of third scenario & $72.8 \%$ & $72.1 \%$ & $63.8 \%$ & $59.3 \%$ \\
Average energy gap (second scenario) $\left[k_{\mathrm{B}} T\right]$ & 0.34 & 0.32 & 0.33 & 0.34 \\
Average energy gap (third scenario) $\left[k_{\mathrm{B}} T\right]$ & 0.92 & 0.69 & 0.57 & 0.54 \\
\hline \hline
\end{tabular}


TABLE IV: The ratio of invariant groundstates of the reference potential under the application of variant potentials

\begin{tabular}{llcc}
\hline \hline & Additive96 & MJ85 & Additive85 \\
\hline Second Scenario & $10.5 \%$ & $35.8 \%$ & $9.0 \%$ \\
Third Scenario & $33.0 \%$ & $43.4 \%$ & $28.5 \%$ \\
Total $^{a}$ & $24.5 \%$ & $40.4 \%$ & $21.0 \%$ \\
\hline \hline
\end{tabular}

\footnotetext{
${ }^{a}$ The total probability is not simply the sum of above numbers but their average with respect to the weights given in table
}

\section{Figure captions:}

- Figure [1: The reference potential (MJ96) and the extracted additive potential (Additive96) parameters are compared with each other, showing a strong correlation $(r=0.982)$.

- Figure 2]: Graphical comparison of the reference potential (left) and extracted additive potential (right). Darker elements correspond to stronger interactions.

- Figure 3]: The histogram of the elements of $D$ (the difference of Additive96 and MJ96 matrices). Except a few elements, the rest of the differences are less then $0.5 k_{\mathrm{B}} T$.

- Figure 4]: Color online: The number of highly compact structures, their corresponding contact vectors and the number of vectors with $g=1$ for different lengths are presented in a log-log plot.

- Figure [5]: The designability of a given structure in the reference model (MJ96) is compared with its designability in two other models: (a) Additive96 (b) MJ85. The most designable structures are the same in both cases.

- Figure [6]: (a) The histogram of difference of designabilities of a given structure evaluated by Additive96, $n_{s}^{\prime}$, and MJ96, $n_{s}$. (b) The histogram of the relative change in the designabilities. The difference in designabilities can be neglected, compared to the designabilities themselves. 
- Figure [7]: Color online: The structures are sorted in descending order of their designabilities in different models. (a) Designability almost is a log-linear function of rank in all studied models. (b) The same plot in a linear-linear scale. The vertical axis is shifted by mean of designability of the structures. The horizontal axis also scaled by the total number of structures. All of the graphs pass through a fixed point.

- Figure 8]: The average energy gap between the groundstate and the first excited state of a given structure is compared to its designability in different models. (a) $4 \times 4$ structures in the MJ96 potential. (b) $4 \times 4$ structures in the Additive96 potential. (c) $6 \times 6$ structures in the MJ96 potential. (d) $6 \times 6$ structures in the Additive96 potential. In the case of additive models ( $b$ and $d$ ), the structures with $g>1$ (degenerate groundstate, $\Delta=0$ ) have been ignored. A jump is observed in subfigures (a) and (b) which is associated to the finite size effect (see text).

- Figure [9]: The probability of finding the same groundstate for a given sequence using both Additve96 and MJ96 models vs. the energy gap in MJ96 model $(\Delta)$.

- Figure [10]: The histogram of the change in the energy gap of sequences in the reference $(\Delta)$ and the additive $\left(\Delta^{\prime}\right)$ models. 


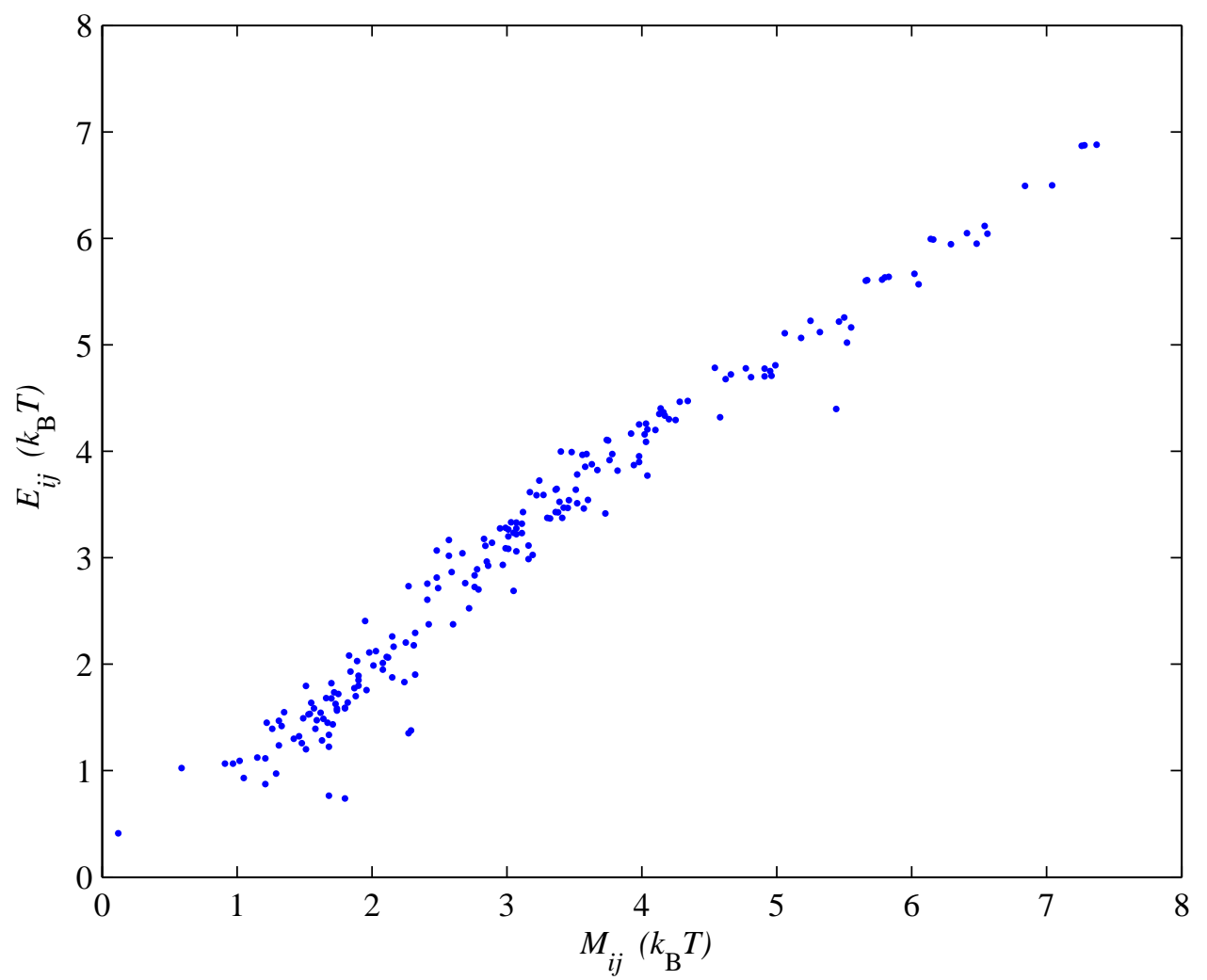

FIG. 1:
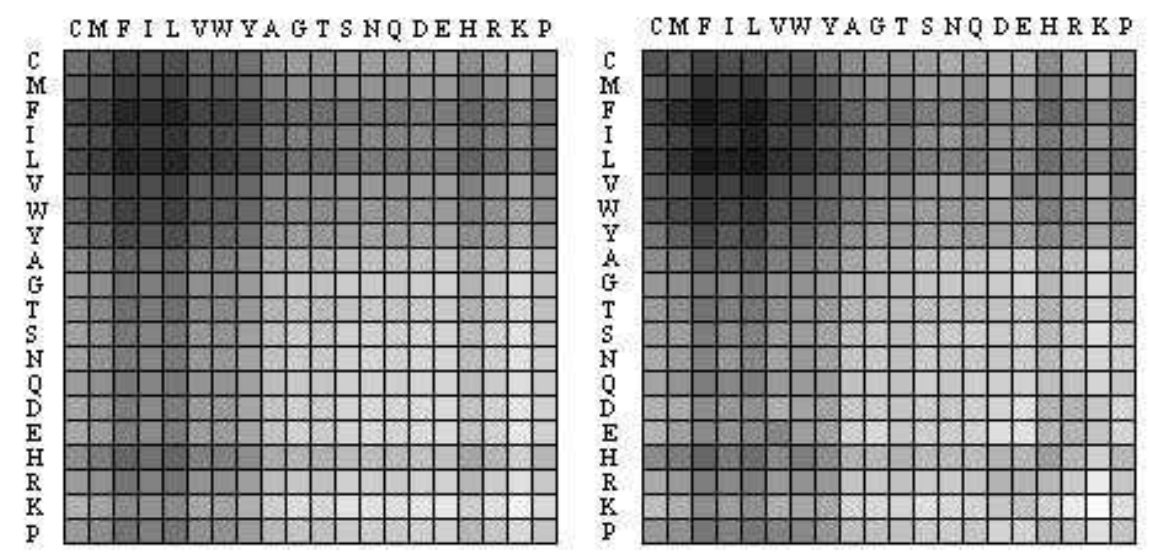

FIG. 2: 


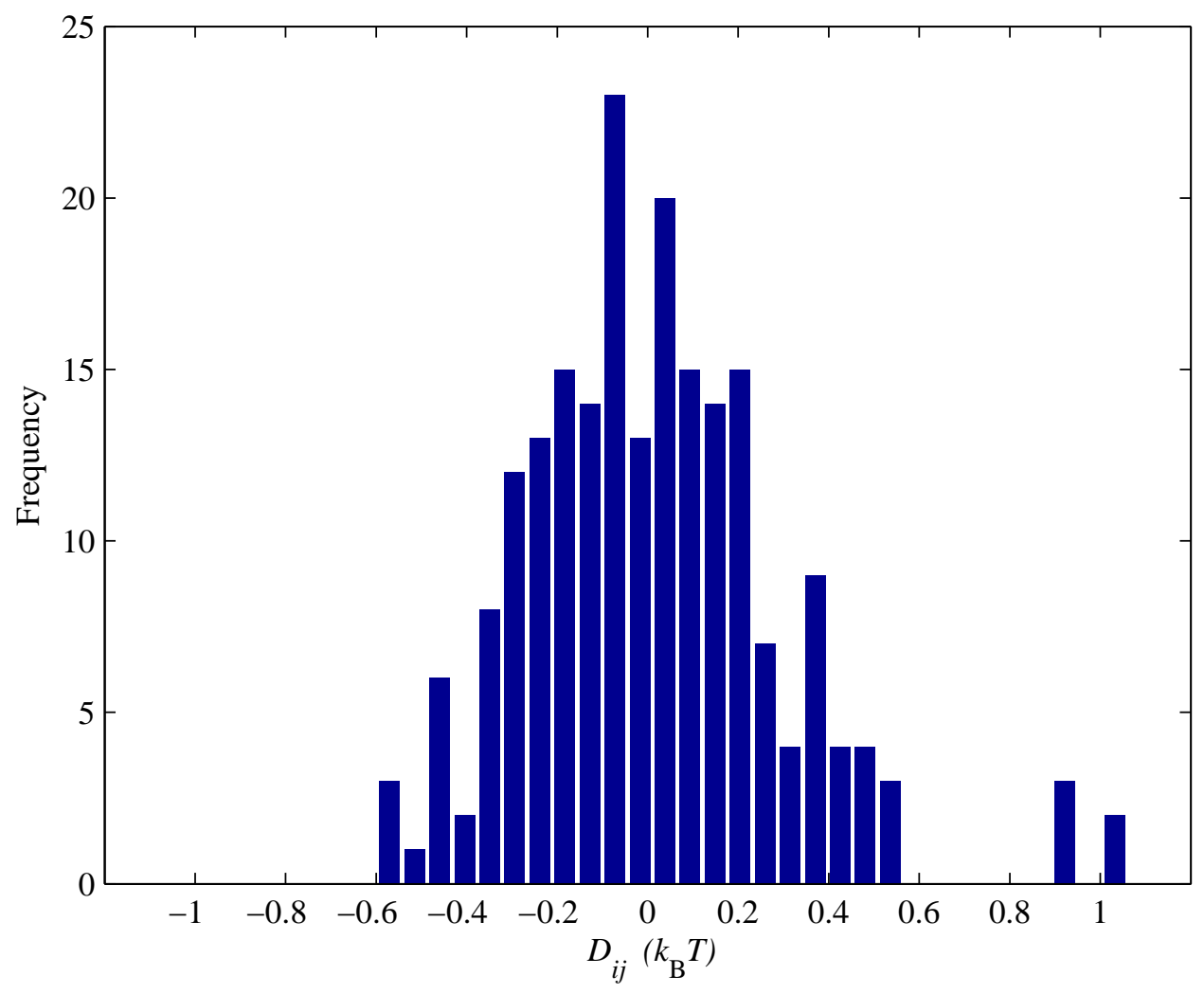

FIG. 3: 


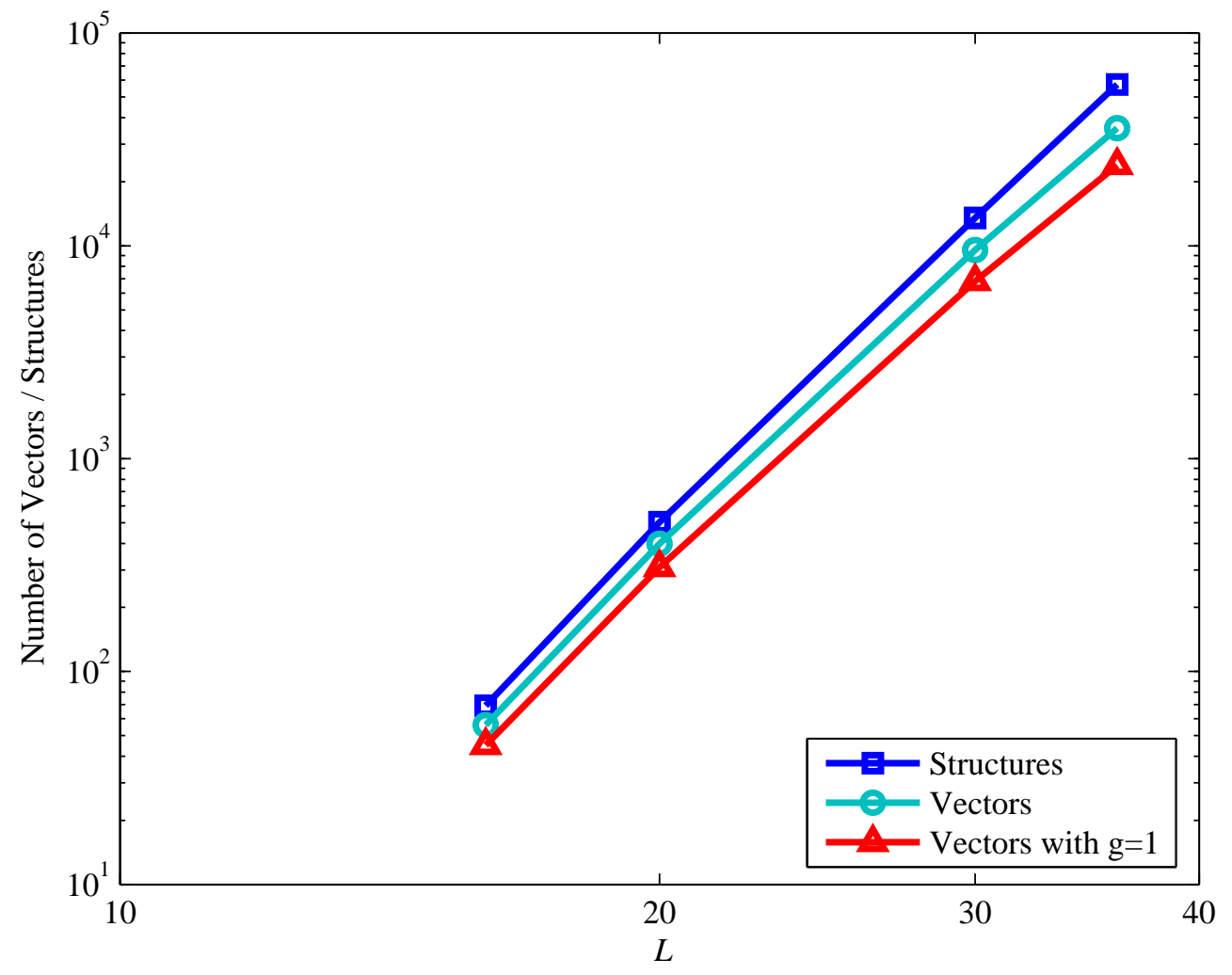

FIG. 4: 

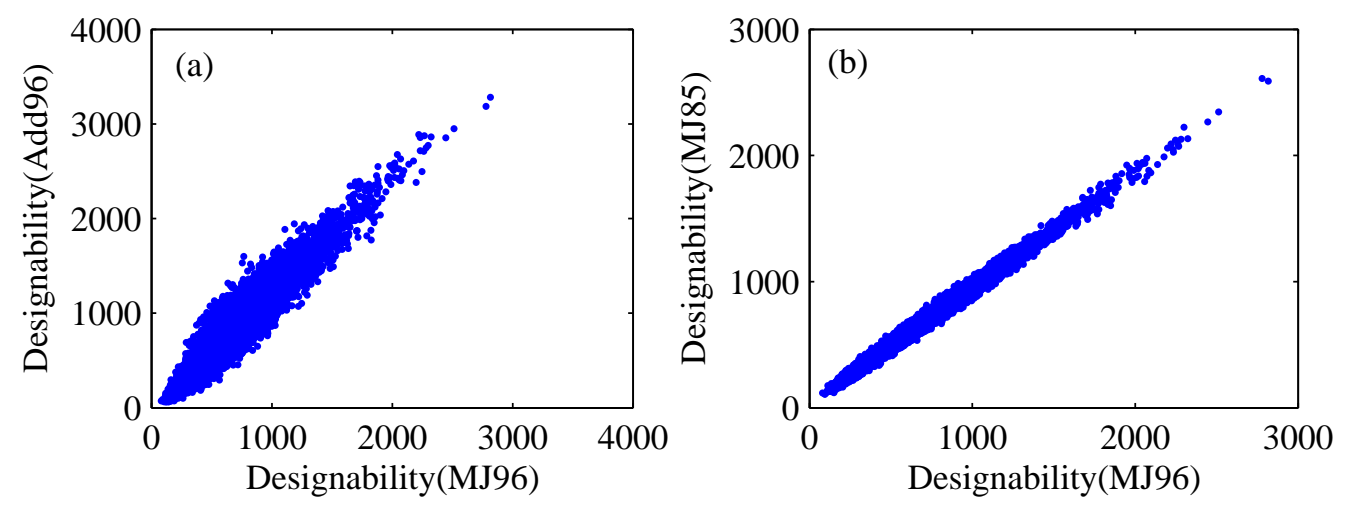

FIG. 5: 

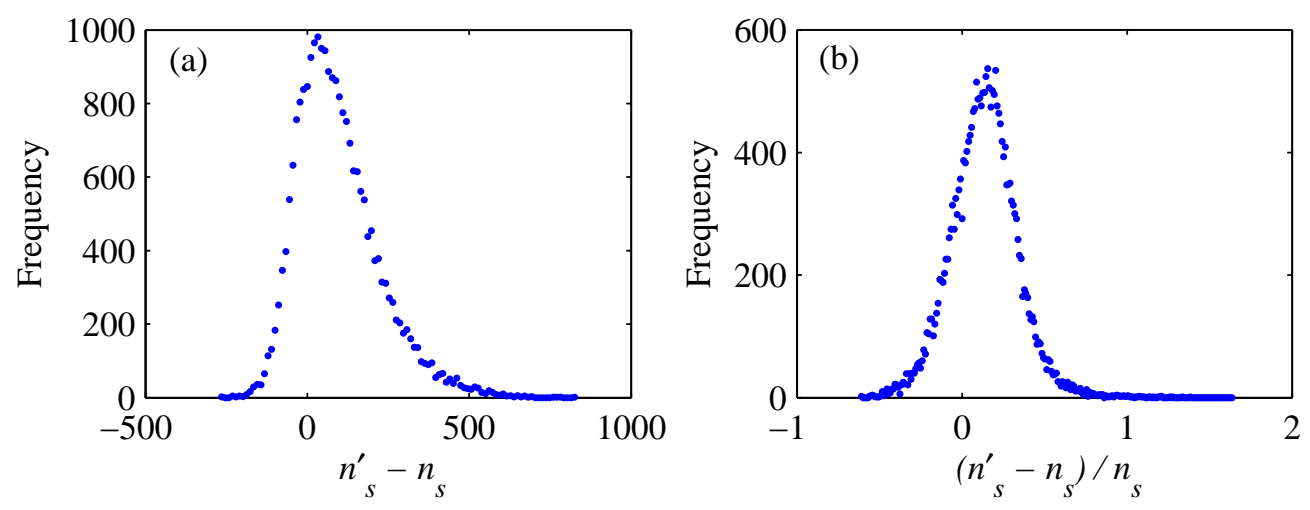

FIG. 6: 

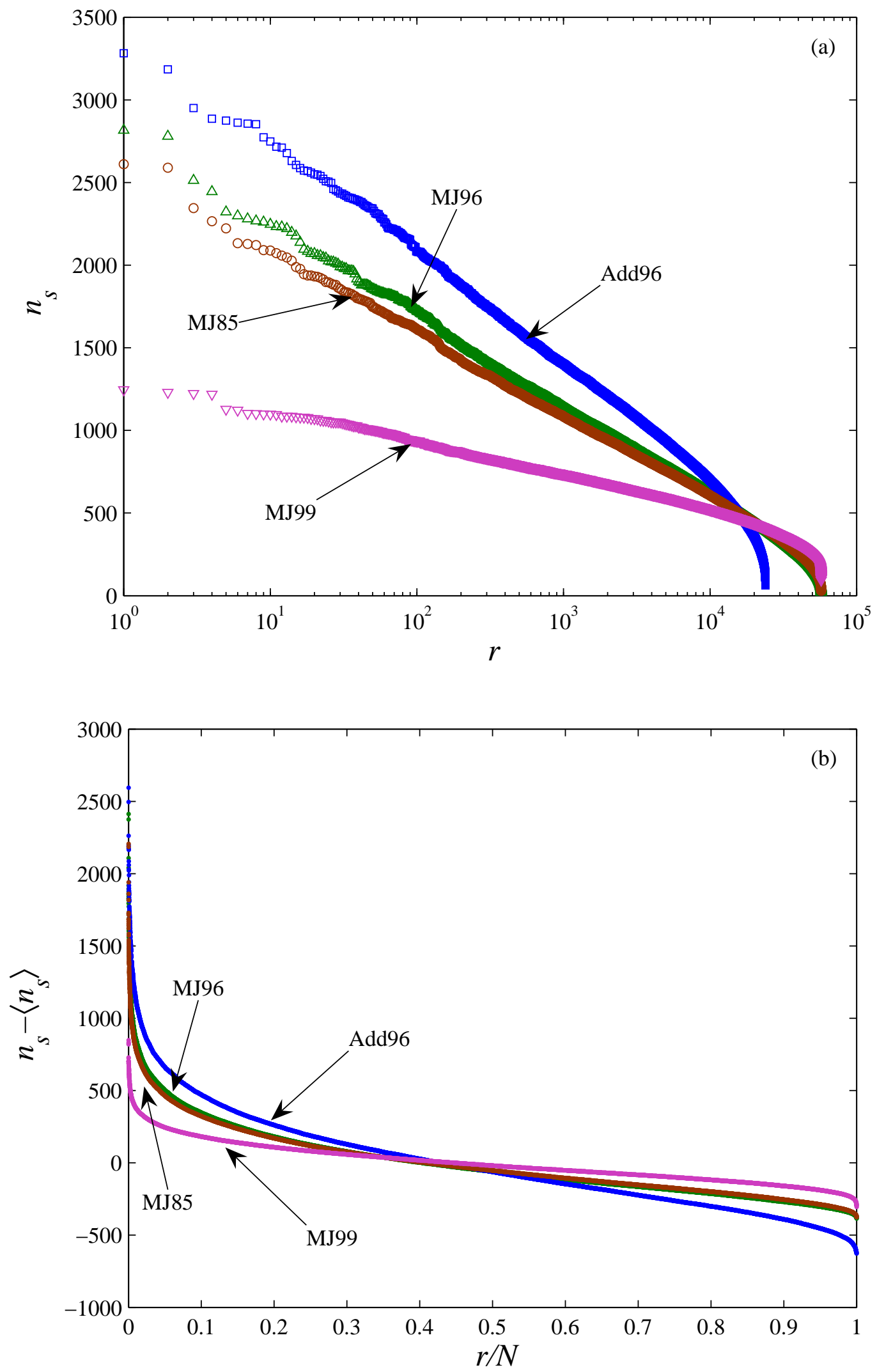

FIG. 7: 

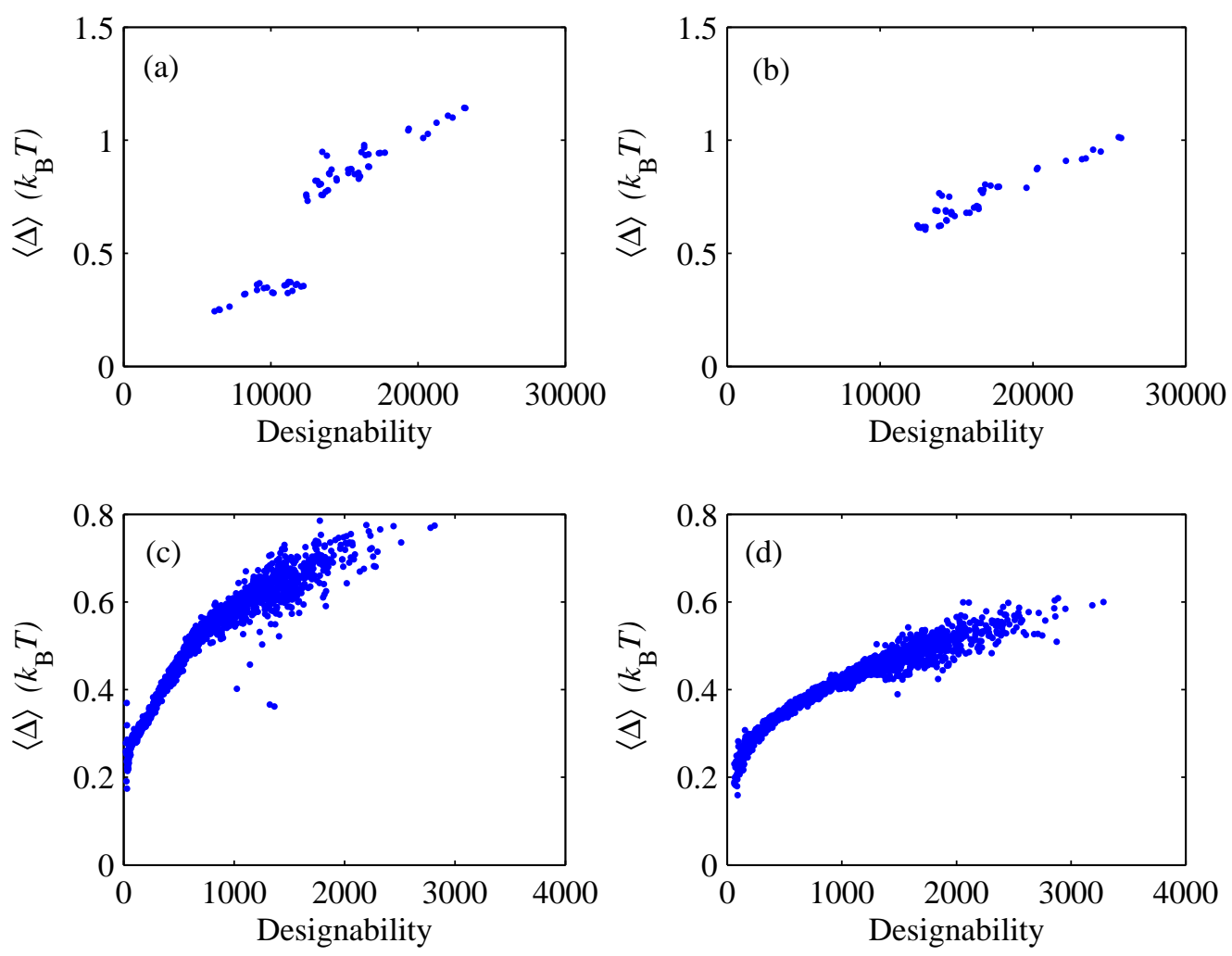

FIG. 8: 


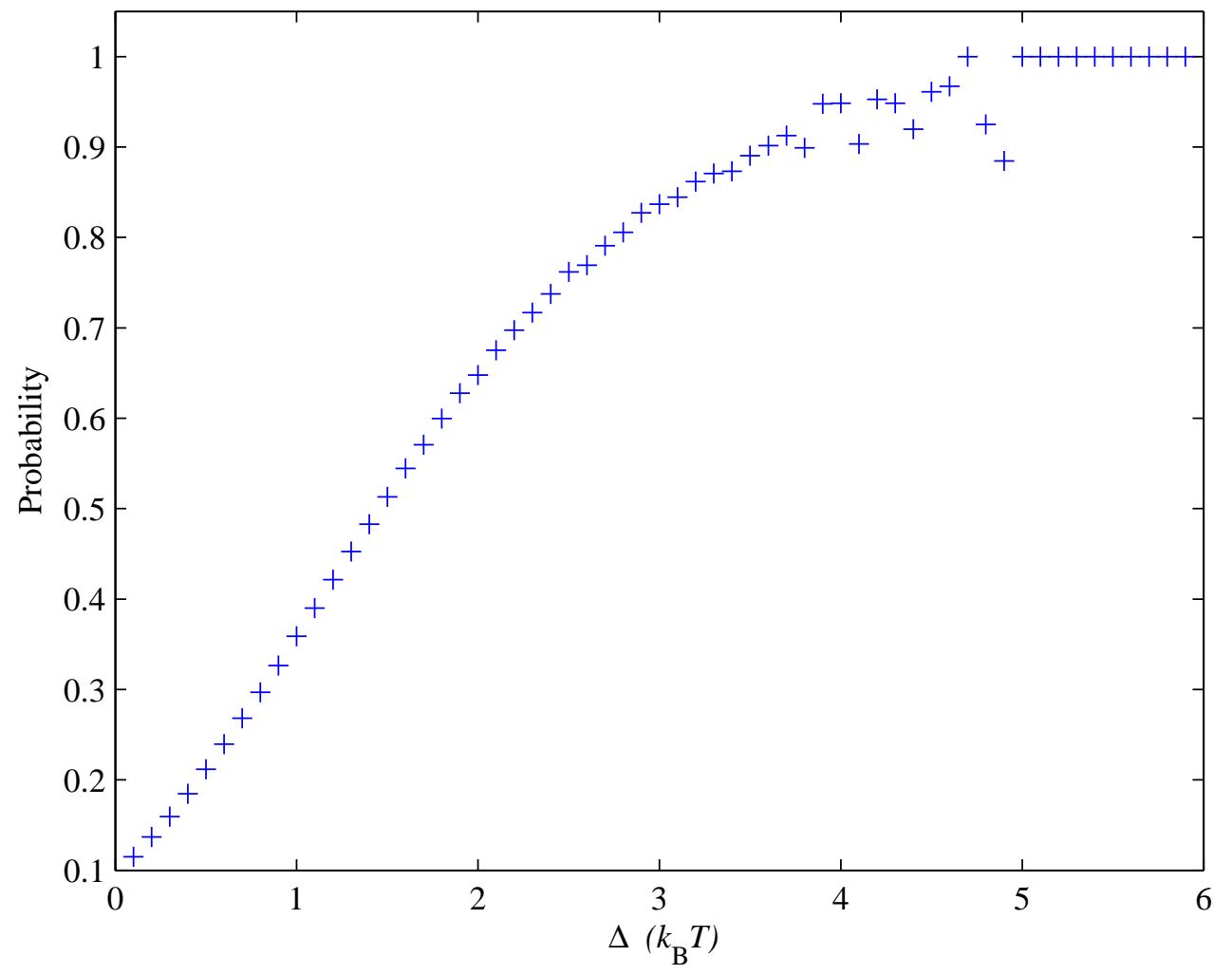

FIG. 9: 


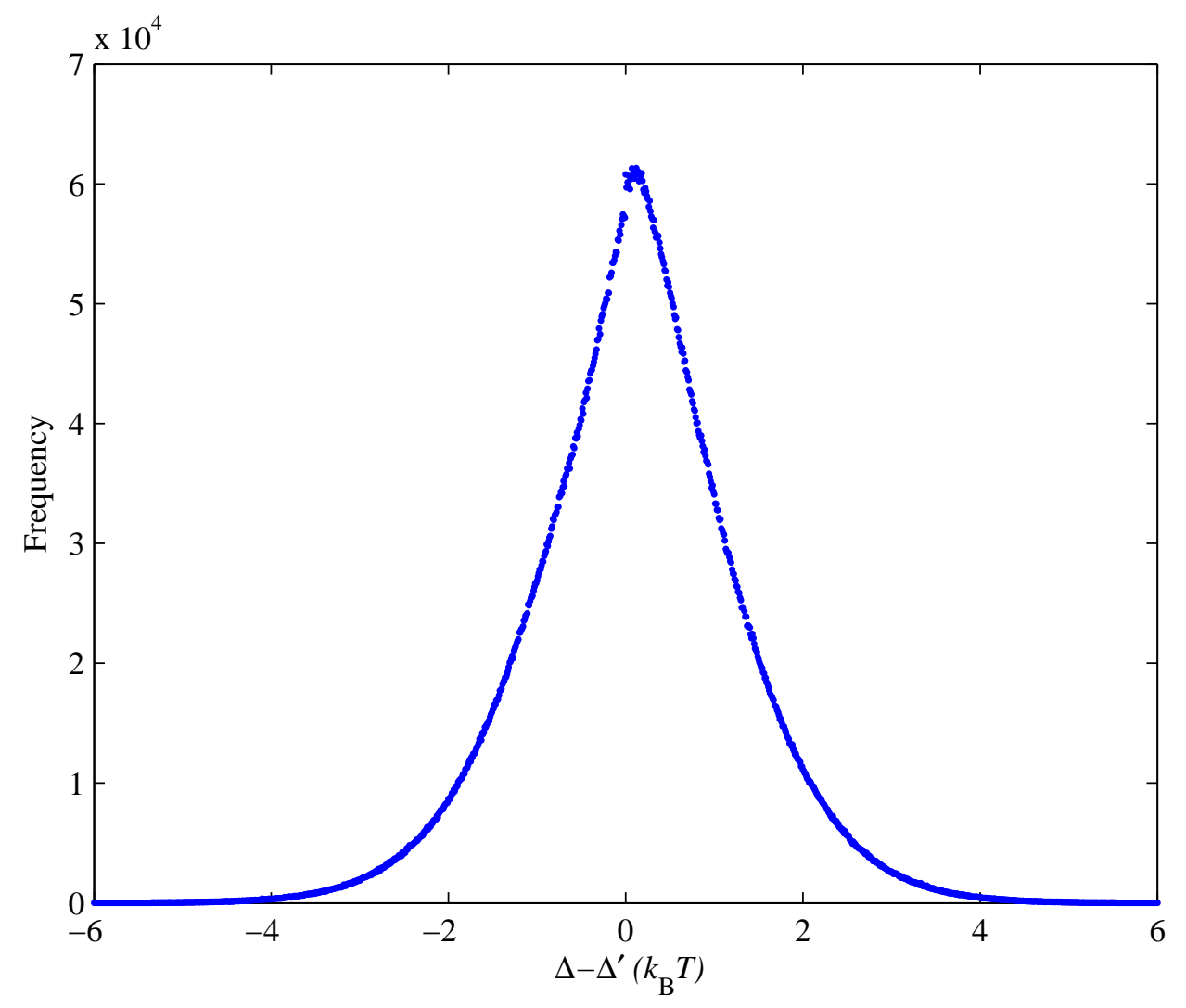

FIG. 10: 